Pacific Journal of Mathematics

ASYMPTOTIC RELATIONS BETWEEN PERTURBED LINEAR
SYSTEMS OF ORDINARY DIFFERENTIAL EQUATIONS 


\title{
ASYMPTOTIC RELATIONS BETWEEN PERTURBED LINEAR SYSTEMS OF ORDINARY DIFFERENTIAL EQUATIONS
}

\author{
Thomas G. Hallam and Nelson OnUChic
}

A generalization of the concept of asymptotic equivalence of two systems of ordinary differential equations is investigated. This extension of asymptotic equivalence is novel in two ways. First, the dimensions of the linear asymptotic subspaces of the differential equations are utilized. Secondly, the two Banach spaces $L^{\infty}$ and $L_{0}^{\infty}$, that are implicitly used in the usual definition of asymptotic equivalence, are replaced by two (arbitrary) Banach spaces that are stronger that $L(X)$. The main theorem establishes a functional asymptotic relationship between the solutions of two perturbed linear differential equations that utilizes the above modifications.

Consider the systems of ordinary differential equations

$$
\begin{aligned}
& y^{\prime}=A(t) y+f_{1}(t, y), \\
& x^{\prime}=A(t) x+f_{2}(t, x),
\end{aligned}
$$

where $x$ and $y$ are vectors in an $n$-dimensional vector space $X, A(t)$ is an $n \times n$ matrix defined on $J=[0, \infty)$, and $f_{1}(t, y)$ and $f_{2}(t, x)$ are $n$-dimensional vector functions defined on $J \times X$.

The equations (1) and (2) are said to be asymptotically equivalent if for each bounded solution $y=y(t)$ of (1) there exists a bounded solution $x=x(t)$ of (2) such that

$$
\lim _{t \rightarrow \infty}[x(t)-y(t)]=0 ;
$$

and, conversely, for each bounded solution $x=x(t)$ of (2) there exists a bounded solution $y=y(t)$ of (1) such that (3) holds. If two nonlinear systems are just known to be asymptotically equivalent then very little information can be obtained about the dimensions of the corresponding asymptotic subspaces of solutions. To remedy this situation, we formulate in $\S 2$, a definition that takes advantage of the dimensions of certain linear asymptotic manifolds of solutions of equations (1) and (2). A general Banach space setting for the equivalence is exploited in this new definition.

The principal tool used in this work is a result of P. Hartman and N. Onuchic [6] on the asymptotic integration of ordinary differential equations.

As corollaries to our main theorem, several recent results on the 
asymptotic equivalence of perturbed linear systems are obtained. In particular, we obtain extensions of results in the articles by F. Brauer and J. S. W. Wong [1] and T. G. Hallam [4]. Applications in new directions are also given. Results related to this problem that are found by using the same methods may be found in the papers [8] and [9] by N. Onuchic.

2. Preliminary material and definitions. In this section we state definitions, indicate notation, and summarize the results needed in our development of this problem. Additional details may be found in [6].

The symbol $\|\cdot\|$ will denote a norm on $X$. The symbol $\beta=\beta(J$, $R$ ) denotes a Banach space of real-valued functions defined on $J$ with the norm of $\phi \in \beta$ denoted by $|\phi|_{\beta}$. By $B=\beta(J, X)$ we represent the space of measurable functions $x=x(t)$ defined on $J$ with values in $X$ such that $\|x(t)\| \in \beta$, and with $|x(t)|_{B}=|\|x(t)\||_{\beta}$. We let $L=L(J$, $R$ ) denote the space of locally Lebesgue integrable real-valued functions defined on $J$, with the topology of convergence in the mean of order one on bounded subintervals of $J$, and let $L(X)=L(J, X)$ represent the space of measurable functions $x$ from $J$ to $X$ such that $\|x(t)\| \epsilon$ $L(J, R)$. A Banach space $B$ is stronger than $L(X)$ if $B$ is algebraically contained in $L(X)$ and convergence in $B$ implies convergence in $L(X)$. Every Banach space of measurable functions from $J$ to $X$ used below will be tacitly assumed to be stronger than $L(X)$.

The class $H=H(R)$ consists of all Banach spaces $\beta=\beta(J, R)$ of measurable functions from $J$ to $R$ with the four properties

(i) $\beta$ is stronger than $L(J, R)$;

(ii) if $\phi \in \beta, \psi$ is measurable, and $|\psi(t)| \leqq|\phi(t)|$, then $\psi \in \beta$ and $|\psi|_{\beta} \leqq|\phi|_{\beta}$

(iii) if $h_{I}$ is the characteristic function of the interval $I$, then $h_{I} \in \beta$ for all intervals $I=[0, T]$, for $T>0$;

(iv) $\beta$ is lean at infinity; that is, if $\phi \in \beta$, then $h_{[0, r]} \phi \rightarrow \phi$ as $T \rightarrow \infty$.

For example, the spaces $L^{p}(J, R), 1 \leqq p<\infty$, are contained in $H(R)$. The same is true of $L_{0}^{\infty}(J, R)$, the subspace of $L^{\infty}(J, R)$ whose elements $x$ satisfy the condition ess $\lim _{t \rightarrow \infty} x(t)=0$. However, $L^{\infty}(J, R)$ itself is not contained in $H(R)$.

Another useful class of Banach spaces in $H(R)$ consists of spaces $\beta$ defined in the following manner. Let $\psi=\psi(t)>0$ be a measurable function on $J$ such that $\psi$ and $1 / \psi$, are locally bounded on $J$. The space $\beta=L_{\psi, 0}(J, R)$ contains all measurable functions $\phi=\dot{\phi}(t)$ such that $\phi / \psi \in L_{0}^{\infty}(J, R)$ with $|\phi|_{\beta}=|\phi / \psi|_{L^{\infty}}$.

We represent by $H(X)$ the class of all Banach spaces $B=\beta(J$, $X)$ where $\beta(J, R)$ is in $H(R)$. 


\section{In the equations}

$$
\begin{gathered}
z^{\prime}=A(t) z, \\
w^{\prime}=A(t) w+b(t),
\end{gathered}
$$

$A(t)$ is a locally Lebesgue integrable $n \times n$ matrix defined on $J$, and $b \in L(X)$.

We assume that the Banach spaces in the sequel consist of measurable functions from $J$ to $X$ unless the contrary is specified. For such a Banach space $D$, let $X_{0 D}$ denote the set of initial points $x(0) \epsilon$ $X$ of solutions $x=x(t)$ of equation (4) which are in $D$. Let $X_{1 D}$ be any subspace of $X$ complementary to $X_{0 D}$ and $P_{0 D}$ the projection of $X$ onto $X_{0 D}$ which annihilates $X_{1 D}$. A pair of Banach spaces $(B, D)$ is called admissible for $A(t)$ if for every $b \in B$, (5) has at least one solution $w=w(t)$ in $D$. A function $x=x(t)$ from $[T, \infty)$ to $X, T \geqq$ 0 , is asymptotically in the Banach space $B$ if there is a function $\widetilde{x}=$ $\widetilde{x}(t)$ from $J$ to $X$ which is in $B$ and such that $\widetilde{x}(t)=x(t)$ for $t \geqq T$. Whenever the function $x$ is asymptotically in $B$ and $x$ is also a solution of a differential equation then we will say that $x$ is an asymptotic $B$ solution of the differential equation.

Let $p$ and $q$ be integers that satisfy the inequalities $0 \leqq p \leqq n$, $0 \leqq q \leqq n$. Equations (1) and (2) are $(p, q)$-asymptotically related with respect to the ordered pair $\left\{D_{1}, D_{2}\right\}$ of Banach spaces if the following two conditions hold:

(i) There exists a family $F_{p}$ of asymptotic $D_{1}$ solutions of (1) which depends upon at least $p$ parameters.

(ii) For each solution $y=y(t)$ of (1) in $F_{p}$, there corresponds a family $F_{q}$ of solutions $x=x(t)$ of (2), which depends upon at least $q$ parameters, such that $y-x$ is asymptotically in $D_{2}$ for each $x \in F_{q}$.

We adopt the convention that a family which depends upon 0 parameters must consist of at least one member.

If the family of all bounded solutions of (1) is a $p$-parameter family and the family of all bounded solutions of (2) is a $q$-parameter family then the concept of asymptotic equivalence may be formulated as follows: Equations (1) and (2) are $(p, 0)$-asymptotically related with respect to $\left\{L^{\infty}, L_{0}^{\infty}\right\}$ and equations $(2)$ and $(1)$ are $(q, 0)$-asymptotically related with respect to $\left\{L^{\infty}, L_{0}^{\infty}\right\}$.

Let $C=C(X)$ denote the space of continuous functions from $J$ to $X$ with the compact open topology. Let $\Sigma_{D, \rho}$ be the closed ball of radius $\rho$ in $D$ and let $S_{D, \rho}=\Sigma_{D, \rho} \cap C$ and $\bar{S}_{D, \rho}$ be the closure of $S_{D, \rho}$ in $C(X)$.

A basic requirement imposed upon the functions $f_{i}$ of (1) and (2) is 
(6) $\quad f_{i}(t, x)(i=1,2)$ is a measurable function of $t$ for fixed $x \in X$ and a continuous function of $x$ for fixed $t \in J$. Furthermore, the continuity of $f_{i}$ in $x$ is uniform for $t$ in compact subintervals of $J$.

The matrix $A(t)$ will always satisfy the condition $A(t)$ is locally Lebesgue integrable on $J$.

The next lemma is a consequence of Theorem 1.1 of [6].

LEMma. Suppose that equation (1) has the following properties.

(i) Condition (6) is satisfied for the function $f_{1}$ and condition (7) is satisfied.

(ii) The Banach space $B=\beta(J, X)$ is in $H(X)$ and the pair (B, $D)$ is admissible for $A(t)$.

(iii) There exists a constant $\rho>0$ and a function $r_{\rho}=r_{\rho}(t) \epsilon$ $\beta(J, R)$ such that

$$
\left\|f_{1}(t, y(t))\right\| \leqq r_{\rho}(t)
$$

for all $t \in J$ and all $y \in \bar{S}_{D, \rho}$.

Let $\xi_{0} \in X_{0 D}$. Then, there exist positive constants $C_{0}, K$ depending only upon $A(t), B, D, X_{1 D}$ (but not on $f_{1}$ nor $\xi_{0}$ ) such that whenever $\left\|\xi_{0}\right\|$ is sufficiently small and $T$ is sufficiently large so that

$$
C_{0}\left\|\xi_{0}\right\|+K\left|h_{[T, \infty)} r_{\rho}\right|_{\beta} \leqq \rho,
$$

then (1) has an asymptotic $D$ solution $y=y(t)$ valid on $[T, \infty)$ with the properties

(iv) $P_{0 D} Z^{-1}(T) y(T)=\xi_{0}$, where $Z(t)$ is the fundamental matrix of (4) with $Z(0)=I_{n}$; and

(v) The solution $y$ has an extension $\widetilde{y}$ which is valid on $J$ and is a solution of

$$
y^{\prime}=A(t) y+h_{[T, \infty)}(t) f_{1}(t, y)
$$

such that $\widetilde{y}(T)=y(T)$ and $|\widetilde{y}(t)|_{D} \leqq \rho$.

Proof. The only hypotheses of Theorem 1.1 of [6] which are not explicitly given aboven above are the conditions denoted by (b) and (c) in [6]. For (9), condition (b) states that the transformation

$$
y(t) \rightarrow \widetilde{f}(t, y(t)) \equiv h_{[T, \infty)}(t) f_{1}(t, y(t))
$$

is a continuous map of the subset $\bar{S}_{D, \rho}$ of $C(X)$ into $B$. This is a consequence of the facts that $B \in H(X)$ and $f_{1}$ satisfies conditions (6) 
and (8). To establish this, let $\varepsilon>0$ be given; then $\tau \geqq 0$ can be chosen sufficiently large so that $\left|h_{[\tau, \infty)}(t) r_{\rho}(t)\right|_{\beta}<\varepsilon / 4$. By virtue of (6), there exists a $\delta=\delta(\varepsilon, \tau)>0$ such that if $y_{1}, y_{2} \in \bar{S}_{D, \rho}$ and $\mid y_{1}(t)-$ $y_{2}(t) \mid<\delta$ for $t \in[0, \tau]$ then

$$
\left|h_{[0, \tau]}(t)\left[\tilde{f}_{1}\left(t, y_{1}(t)\right)-\tilde{f}_{1}\left(t, y_{2}(t)\right)\right]\right|_{B}<\varepsilon / 2 .
$$

Therefore, if $y_{1}$ and $y_{2}$ are in $\bar{S}_{D, \rho}$ with

$$
\left|h_{[0, \tau]}(t)\left[y_{1}(t)-y_{2}(t)\right]\right|<\delta,
$$

then

$$
\begin{aligned}
\left|\widetilde{f}_{1}\left(t, y_{1}(t)\right)-\widetilde{f}_{1}\left(t, y_{2}(t)\right)\right|_{B} \leqq & \left|h_{[0, \tau]}(t)\left[\widetilde{f}_{1}\left(t, y_{1}(t)\right)-\widetilde{f}_{1}\left(t, y_{2}(t)\right)\right]\right|_{B} \\
& +\left|h_{[\varepsilon, \infty)}(t) \widetilde{f}_{1}\left(t, y_{1}(t)\right)\right|_{B} \\
& +\left|h_{[\tau, \infty)}(t) \widetilde{f}_{1}\left(t, y_{2}(t)\right)\right|_{B} \\
& <\varepsilon / 2+2\left|h_{[\tau, \epsilon)}(t) r_{\rho}(t)\right|_{\beta} \\
& <\varepsilon .
\end{aligned}
$$

This shows that (b) is satisfied.

For (9), condition (c) states that there exists a constant $r>0$ such that $\left|\widetilde{f}_{1}(t, x(t))\right|_{B} \leqq r$ for $x(t) \in \bar{S}_{D, \rho}$. This follows by taking $r=\left|h_{[T, \infty)} r_{\rho}\right|_{\beta}$.

Theorem 1.1 of [6] may now be applied to system (9) to establish the existence of a $D$ solution $\widetilde{y}=\widetilde{y}(t)$ of (9) which satisfies $|\widetilde{y}(t)|_{D} \leqq$ $\rho$ and $P_{0 D} \widetilde{y}(0)=\xi_{0}$. Since $\widetilde{f}_{1}(t, y)=0$ for $0 \leqq t<T$, it follows that $\widetilde{y}(T)=Z(T) \widetilde{y}(0)$ and hence

$$
P_{0 D} \widetilde{y}(0)=P_{0 D} Z^{-1}(T) \widetilde{y}(T)=\xi_{0} .
$$

The function $y(t)=\widetilde{y}(t), t \geqq T$, is an asymptotic $D$ solution such that $P_{0 D} Z^{-1}(T) y(T)=\xi_{0}$. This completes the proof of the lemma.

REMARK 1. If $m=\operatorname{dim} X_{0 D}$, this lemma implies the existence of a family of asymptotic $D$ solutions of (1) which depends at least upon $m$ parameters.

REMARK 2. Condition (iii) of the Lemma is sometimes difficult to establish. In the instance where $D=L^{\infty}$ or $D=L_{0}^{\infty}$, a simple sufficient condition for (iii) is that there exists a $\lambda_{\rho}=\lambda_{\rho}(t)$ in $\beta(J, R)$ such that $\|f(t, x)\| \leqq \lambda_{\rho}(t)(t \in J ;\|x\| \leqq \rho)$. A sufficient condition for (iii), without specifying $D$, is that there exists a $\lambda=\lambda(t) \in \beta(J, R)$ such that $\|f(t, x)\| \leqq \lambda(t)(t \in J ; x \in X)$. Theorem 4 below gives a sufficient condition for inequality (8) to hold whenever $B$ and $D$ are certain spaces of $L^{p}$ type. 
3. Asymptotic relations. This section contains our main result and some applications.

THEOREM 1. Suppose that equations (1) and (2) satisfy the following conditions.

(i) Assumptions (6) and (7) hold.

(ii) The space $B_{i}=\beta_{i}(J, X)$ is in $H(X)$ and the pair $\left(B_{i}, D_{i}\right)$ is admissible, $i=1,2$.

(iii) There exist constants $\rho_{i}>0$ and functions

$$
r_{\rho_{i}} \equiv r_{\rho_{i}}(t) \in \beta_{i}(J, R)
$$

such that

$$
\| f_{1}\left(t, y(t) \| \leqq r_{\rho_{1}}(t)\right.
$$

for all $t \in J$ and all $y \in{\overline{S_{D_{1}}, \rho_{1}}}_{\text {; }}$ and

$$
\left\|f_{2}(t, u(t)+y(t))-f_{1}(t, y(t))\right\| \leqq r_{\rho_{2}}(t)
$$

for all $t \in J$, all $y \in \bar{S}_{1 D, \rho_{1}}$, and all $u \in \bar{S}_{D_{2}, \rho_{2}}$.

(iv) The dimensions of $X_{0 D_{1}}$ and $X_{0 D_{2}}$ are $p$ and $q$ respectively. Then, under these hypotheses, equations (1) and (2) are $(p, q)$-asymptotically related with respect to $\left\{D_{1}, D_{2}\right\}$.

Proof. The existence of a family $F_{p}$ of asymptotic $D_{1}$ solutions of (1), which depends upon at least $p$ parameters, is an immediate consequence of the Lemma. Let $y=y(t)$ be a solution of (9) where $T$ and $y \in F_{p}$ are as given by the Lemma. It follows that $|y|_{D_{1}} \leqq \rho_{1}$.

The change of variable $u=x-y(t)$ leads to the differential equation

$$
u^{\prime}=A(t) u+f(t, u) \quad(t \geqq T ; u \in X)
$$

where

$$
f(t, u)=f_{2}(t, u+y(t))-f_{1}(t, y(t)) .
$$

It is convenient to consider equation (12) as

$$
u^{\prime}=A(t) u+h_{[T, \infty)}(t) f(t, u)
$$

From (11) it follows that for all $u \in \bar{S}_{D_{2}, \rho_{2}}$,

$$
\left\|h_{[T, \infty)}(t) f(t, u(t))\right\| \leqq h_{[T, \infty)}(t) r_{\rho_{2}}(t),
$$

The lemma now implies that there exists a family $F_{q}$ of asymptotic $D_{2}$ solutions of (12) that depends upon at least $q$ parameters. For each $u \in F_{q}, x(t)=u(t)+y(t)$ is a solution of equation (2); hence, there 
is a family $G_{q}$ of solutions of (2), which depends at least upon $q$ parameters, such that $x-y$ is asymptotically in $D_{2}$ for each $x \in G_{q}$. This completes the proof of Theorem 1 .

CoRollary 1. Suppose that conditions (i), (ii), and (iv) of Theorem 1 are satisfied for the spaces $B_{i}=B=\beta(J, X) \in H(X), i=1,2$. In addition, suppose that there exists $a \lambda=\lambda(t) \in \beta(J, R)$ such that $\| f_{i}(t$, $x) \| \leqq \lambda(t)$ for all $t \in J$ and all $x \in X, i=1,2$. Then, equations (1) and (2) are $(p, q)$-asymptotically related with respect to $\left\{D_{1}, D_{2}\right\}$ and equations (2) and (1) are $(q, p)$-asymptotically related with respect to $\left\{D_{2}, D_{1}\right\}$.

Proof. Taking into account Remark 2, we have that (10) is satisfied with $r_{\rho_{1}}=\lambda$ and (11) is satisfied with $r_{\rho_{2}}=2 \lambda$. Since the hypotheses are symmetric for both the cases $i=1,2$, Theorem 1 yields the desired conclusion.

For the particular case in which $D_{1}=L^{\infty}$ and $D_{2}=L_{0}^{\infty}$ we obtain the following results.

THEOREM 2. Suppose that equations (1) and (2) satisfy the following conditions

(i) Assumption (i) of Theorem 1 holds.

(ii) The function $V(t, r)$ is nonnegative for $(t, r) \in J \times J$, nondecreasing in $r$ for fixed $t$, and

$$
\left\|f_{i}(t, x)\right\| \leqq V(t,\|x\|),(i=1,2 ; t \in J ; x \in X) .
$$

(iii) The space $B=\beta(J, X)$ is in $H(X) ; V(t, r)$ is in $\beta(J, R)$ for each fixed $r>0$; and, $\left(B, L_{0}^{\infty}\right)$ is admissible for $A(t)$.

(iv) The dimensions of $X_{0 L^{\infty}}$ and $X_{0 L_{0}^{\infty}}$ are $p$ and $q$ respectively. Then, under these hypotheses, equations (1) and (2) are $(p, q)$-asymptotically related with respect to $\left\{L^{\infty}, L_{0}^{\infty}\right\}$.

Proof. Theorem 2 is a consequence of Theorem 1 provided we show that the inequality (14) implies that the inequalities (10) and (11) hold. Let $\rho_{i}>0(i=1,2)$ be given. For all $y \in \bar{S}_{L^{\infty}, \rho_{1}}$,

$$
\left\|f_{1}(t, y(t))\right\| \leqq V\left(t, \rho_{1}\right)
$$

therefore, (10) is satisfied because $V\left(t, \rho_{1}\right)$ is in $\beta(J, R)$. To see that (11) holds, we only need observe that for all $u \in \bar{S}_{L_{0}^{\infty}, \rho_{2}}$ and all $y \in \bar{S}_{L^{\infty}, \rho_{1}}$

$$
\left\|f_{2}(t, u(t)+y(t))-f_{1}(t, y(t))\right\| \leqq 2 V\left(t, \rho_{1}+\rho_{2}\right) .
$$

As Corollaries to the above theorem, we obtain extensions of some results of Brauer and Wong [1, Theorem 1] and Hallam [4, 
Theorem 2].

COROLlaRY 2. Suppose that equations (1) and (2) satisfy the following conditions.

(i) There exist supplementary projections $P_{1}, P_{2}$ and a constant $K>0$ such that

$$
\begin{aligned}
& \left\|Z(t) P_{1} Z^{-1}(s)\right\| \leqq K, 0 \leqq s \leqq t \\
& \left\|Z(t) P_{2} Z^{-1}(s)\right\| \leqq K, 0 \leqq t \leqq s
\end{aligned}
$$

(ii) The function $f_{1} \equiv 0, f_{2}$ satisfies (6) and

$$
\left\|f_{2}(t, x)\right\| \leqq V(t,\|x\|),(t \in J ; x \in X)
$$

where $V(t, r)$ satisfies hypothesis (ii) of Theorem 2.

(iii) $\int_{0}^{\infty} V(t, r) d t<\infty$ for all $r>0$.

(iv) Assumption (7) and condition (iv) of Theorem 2 hold. Then, under these hypotheses, the equations (1) and (2) are ( $p, q)$-asymptotically related with respect to $\left\{L^{\infty}, L_{0}^{\infty}\right\}$.

Proof. It is known [7, p. 331] that if $\left(L^{1}, L^{\infty}\right)$ is admissible for $A(t)$ then $\left(L^{1}, L_{0}^{\infty}\right)$ is also admissible for $A(t)$. It is also known that $\left(L^{1}, L^{\infty}\right)$ is admissible if and only if (15) is satisfied. The corollary now follows from Theorem 2 with $B=L^{1}$.

COROLlaRY 3. Suppose that the assumptions (ii) and (iv) of Corollary 2 hold. Suppose that there exist sypplementary projections $P_{1}, P_{2}$ and a constant $K>0$ such that

$$
\begin{aligned}
\rho(t) \equiv & \left(\int_{0}^{t}\left\|Z(t) P_{1} Z^{-1}(s)\right\|^{\tau} d s\right)^{1 / \tau} \\
+ & \left(\int_{t}^{\infty}\left\|Z(t) P_{2} Z^{-1}(s)\right\|^{\tau} d s\right)^{1 / \tau} \leqq K, \\
& (t \in J ; 1<\tau<\infty) .
\end{aligned}
$$

Let $\int_{0}^{\infty}[V(t, r)]^{\sigma} d t<\infty$, for all $r>0$, where $\sigma^{-1}+\tau^{-1}=1$. Then, equations (1) and (2) are $(p, q)$-asymptotically related with respect to $\left\{L_{0}^{\infty}, L_{0}^{\infty}\right\}$.

Proof. Conti [2, Theorem 1] has shown that condition (17) is necessary and sufficient for the pair $\left(L^{\sigma}, L^{\infty}\right)$ to be admissible for $A(t)$. However, condition (17) is equivalent to the stronger result that $\left(L^{\sigma}, L_{0}^{\infty}\right)$ is admissible for $A(t)$. To verify this statement, we note that (17), $b \in L^{\sigma}$, and the Hölder inequality imply that 


$$
w(t)=\int_{0}^{t} Z(t) P_{1} Z^{-1}(s) b(s) d s-\int_{t}^{\infty} Z(t) P_{2} Z^{-1}(s) b(s) d s
$$

is a solution of (5) with $\|w(t)\| \leqq K|b|_{L^{\sigma}}, t \in J$.

It will now be shown that $\lim _{t \rightarrow \infty} w(t)=0$. For a given $\varepsilon>0$, $T_{1}=T_{1}(\varepsilon)$ can be chosen so that

$$
\left|h_{\left.\Gamma T_{1}, \infty\right)} b\right|_{L^{\sigma}}<\varepsilon / 2 K \text {. }
$$

Since $\lim _{t \rightarrow \infty}\left\|Z(t) P_{1}\right\|=0$ (see [4, p. 359]), $T_{2} \geqq T_{1}$ may be chosen so that

$$
\left\|Z(t) P_{1}\right\|<\varepsilon / 2 \int_{0}^{T_{1}}\left\|Z^{-1}(s) b(s)\right\| d s, t \geqq T_{2} .
$$

Therefore, for $t \geqq T_{2}$ it follows from (19) and (20) that

$$
\begin{aligned}
\left\|\int_{0}^{t} Z(t) P_{1} Z^{-1}(s) b(s) d s\right\| & \leqq \int_{0}^{T_{1}}\left\|Z(t) P_{1}\right\|\left\|Z^{-1}(s) b(s)\right\| d s \\
& +\left(\int_{T_{1}}^{t}\left\|Z(t) P_{1} Z^{-1}(s)\right\|^{\tau} d s\right)^{1 / \tau} \\
& \cdot\left(\int_{T_{1}}^{t}\|b(s)\|^{\sigma} d s\right)^{1 / \sigma} \\
& <\varepsilon .
\end{aligned}
$$

This shows that the first term of $w$ in (18) tends to zero as $t$ approaches infinity. The last term in (18) also tends to zero as $t$ approaches infinity since

$$
\left\|\int_{t}^{\infty} Z(t) P_{2} Z^{-1}(s) b(s) d s\right\| \leqq K\left(\int_{t}^{\infty}\|b(s)\|^{\sigma} d s\right)^{1 / \sigma} .
$$

The corollary now follows from Theorem 2.

REMARK 4. The asymptotic equivalence result analogous to Corollary 2 is Theorem 2 of [4]. It yields an $(p, 0)$-asymptotic relation with respect to $\left\{L_{0}^{\infty}, L_{0}^{\infty}\right\}$. It should be noted that we have taken the weight functions $\psi, \phi$ of [4] as $\psi=\phi=1$. The proof that (17) implies $\left(L^{\sigma}, L_{0}^{\infty}\right)$ is admissible for $A(t)$ is essentially contained in [4].

We point out that the statement-equations (1) and (2) are $(p, q)$ asymptotically related with respect to $\{D, D\}$-yields only a natural correspondence between the solutions of (1) and (2). Namely, that correspondence given by $y-x$ is asymptotically in $D$ whenever $y \in D$. This means that $x$ is asymptotically in $D$; hence, the above statement says that (1) $\{(2)\}$ has a family of asymptotic $D$ solutions depending upon at least $p\{q\}$ parameters.

The above corollaries extend known results. The next results are applications of Theorem 1 in a new direction. For this purpose 
consider the Banach space $D=L^{\eta} \cap L_{0}^{\infty}, 1 \leqq \eta<\infty$, where $x \in D$ implies $x \in L^{\eta}$ and $x \in L_{0}^{\infty}$ with $|x|_{D}=\max \left[|x|_{L^{\eta}},|x|_{L_{0}^{\infty}}\right]$; see [7, p. 336]. Later in the paper we will utilize the Banach space $D=L^{\eta} \cap L^{\infty}$ which is defined in an analogous manner.

THEOREM 3. Suppose that equations (1) and (2) satisfy the following conditions.

(i) Assumption (i) of Theorem 1 holds.

(ii) There exist supplementary projections $P_{1}, P_{2}$ and positive constants $\alpha, K$ such that

$$
\begin{array}{ll}
\left\|Z(t) P_{1} Z^{-1}(s)\right\| \leqq K e^{-\alpha(t-s)}, 0 \leqq s \leqq t ; \\
\left\|Z(t) P_{2} Z^{-1}(s)\right\| \leqq K, & 0 \leqq t \leqq s
\end{array}
$$

(iii) There exists a nonnegative function $\lambda=\lambda(t)$ measurable on $J$ such that

$$
\left\|f_{i}(t, x)\right\| \leqq \lambda(t) \quad(i=1,2 ; t \in J ; x \in X)
$$

and

$$
\int^{\infty} t \lambda(t) d t<\infty
$$

(iv) The dimensions of $X_{0 L^{\infty}}, X_{0 L^{r}}(r \geqq 1)$, and $X_{0, D}\left(D=L^{s} \cap L_{0}^{\infty}\right.$, $s \geqq 1$ ) are $p, q$, and $m$ respectively.

Then, under these hypotheses, equations (1) and (2) are $(p, m)$-asymptotically related with respect to $\left\{L^{\infty}, L^{s} \cap L_{0}^{\infty}\right\}$ and equations (2) and (1) are $(q, m)$-asymptotically related with respect to $\left\{L^{r}, L^{s} \cap L_{0}^{\infty}\right\}$.

Proof. Let $\phi=\phi(t)$ be chosen so that $\phi$ is a positive continuous function satisfying

$$
\int^{\infty} t \lambda(t) \phi(t) d t<\infty
$$

and

$$
\lim _{t \rightarrow \infty} \phi(t)=\infty \text {. }
$$

It will be shown that $\left(L_{\psi, 0}, L^{k} \cap L_{0}^{\infty}\right)(k \geqq 1)$ is admissible for $A(t)$ where $\psi=\lambda \phi$.

If $b \in L_{\psi, 0}$, then $w(t)$, as defined by (18), is a solution of (5) since (21) implies that there is a positive constant $K_{1}$ such that

$$
\left\|Z(t) P_{2} Z^{-1}(s) b(s)\right\| \leqq K\|b(s)\| \leqq K_{1} \lambda(s) \phi(s)
$$

where 


$$
\int^{\infty} \lambda(s) \phi(s) d s<\infty
$$

Also, from (21), it follows that

$$
\|w(t)\| \leqq K_{1} \int_{0}^{t} e^{-\alpha(t-s)} \lambda(s) \phi(s) d s+K_{1} \int_{t}^{\infty} \lambda(s) \phi(s) d s .
$$

We obtain from (24) that $w \in L_{0}^{\infty}$ because

$$
\int^{\infty} \lambda(s) \phi(s) d s<\infty
$$

and

$$
\lim _{t \rightarrow \infty} e^{-\alpha t} \int_{0}^{t} e^{\alpha s} \lambda(s) \phi(s) d s=0
$$

(see [3], Lemma 1).

To see that $w=w(t)$ is also in $L^{k}, k \geqq 1$, it is sufficient to show that $w$ is in $L^{1}$ since we have established that $w \in L_{0}^{\infty}$. We note that an integration in (24) leads to

$$
\begin{aligned}
\int_{0}^{t}\|w(\tau)\| d \tau \leqq & K_{1} \int_{0}^{t} d \tau \int_{0}^{\tau} e^{-\alpha(\tau-s)} \lambda(s) \phi(s) d s \\
& +K_{1} \int_{0}^{t} d \tau \int_{\tau}^{\infty} \lambda(s) \phi(s) d s .
\end{aligned}
$$

The integral $\int_{0}^{\infty} d \tau \int_{\tau}^{\infty} \lambda(s) \phi(s) d s$ converges because $\int^{\infty} t \lambda(t) \phi(t) d t$ converges. An integration by parts gives

$$
\begin{aligned}
\int_{0}^{t} e^{-\alpha \tau}\left[\int_{0}^{\tau} e^{\alpha s} \lambda(s) \phi(s) d s\right] d \tau= & -\alpha^{-1} e^{-\alpha t} \int_{0}^{t} e^{\alpha s} \lambda(s) \phi(s) d s \\
& +\alpha^{-1} \int_{0}^{t} \lambda(s) \phi(s) d s .
\end{aligned}
$$

The right side of (26) is bounded which implies that the first integral in the right side of (25) converges as $t \rightarrow \infty$. This implies that $w \in L^{1}$.

The corollary follows from Theorem 1 provided that $\lambda \in L_{\psi, 0}(J, R)$; but, $\lambda / \psi=\phi^{-1}$ and $\lim _{t \rightarrow \infty} \phi^{-1}(t)=0$.

REMARK 5. A sufficient condition for (21) is that $A$ be a constant matrix where all eigenvalues of $A$ with zero real part have linear elementary divisors.

THEOREM 4. Suppose that equations (1) and (2) satisfy the following conditions.

(i) The function $f_{1} \equiv 0 ; f_{2}$ satisfies (6); and (7) is satisfied. 
(ii) There exist positive functions $a=a(t)$ and $b=b(t)$ that are continuous on $J$ and a constant $\delta>0$ such that

$$
\left\|f_{2}(t, x)\right\| \leqq a(t)\|x\|^{\delta}+b(t),(t \in J ; x \in X) .
$$

(iii) The pair $\left(L^{\sigma}, D\right)$ is admissible for $A(t)$, where $1 \leqq \sigma<\infty$, and $D=L^{\eta} \cap L^{\infty}$ with $\sigma \delta \leqq \eta<\infty$.

(iv) The function $a \in L^{\alpha}(J, R)$ where $\alpha=\eta \sigma /(\eta-\sigma \delta)$ and the function $b \in L^{\sigma}(J, R)$.

(v) Let $p=\operatorname{dim} X_{0 L^{\eta}}$ and $q=\operatorname{dim} X_{0 D}$.

Then, under these hypotheses, equations (1) and (2) are $(p, q)$-asymptotically related with respect to $\left\{L^{\eta}, D\right\}$.

Proof. First, we observe that $\bar{S}_{D, \rho}=S_{D, \rho}$. This equality is evident if it is shown that $x \in \bar{S}_{D, \rho}$ implies that $x \in D$ and $|x|_{D} \leqq \rho$. If $x \notin D$ then either $x \notin L^{\eta}$ or $x \notin L^{\infty}$. Since $x \in \bar{S}_{D, \rho}$, there exists a sequence $\left\{x_{n}\right\}$ with $x_{n} \in S_{D, \rho}, n=1,2, \cdots$ such that $\left\{x_{n}\right\}$ converges uniformly to $x$ on compact subintervals of $J$.

Suppose that $x \notin L^{\eta}$; then, corresponding to any $\varepsilon>0$ there is a $T=T(\varepsilon)>0$ such that

$$
\left[\int_{0}^{T}\|x(t)\|^{r} d t\right]^{1 / n}>\rho+\varepsilon .
$$

Corresponding to the positive number $1 / 2 T$, there exists an $N$ such that whenever $n \geqq N$ then $\left\|x_{n}(t)-x(t)\right\|<\varepsilon / 2 T^{1 / \eta}, t \in[0, T]$. Therefore, for $n \geqq N$,

$$
\begin{aligned}
\rho+\varepsilon<\left[\int_{0}^{T}\|x(t)\|^{\eta} d t\right]^{1 / n} \leqq & {\left[\int_{0}^{T}\left\|x_{n}(t)\right\|^{\eta} d t\right]^{1 / n} } \\
& +\left[\int_{0}^{T}\left\|x(t)-x_{n}(t)\right\|^{\eta} d t\right]^{1 / n} \\
\leqq & \rho+\varepsilon / 2 .
\end{aligned}
$$

This contradiction shows that $x \in L^{\eta}$. Because $\left|x_{n}\right|_{L^{\infty}} \leqq \rho$, it is clear that $x \in L^{\infty}$; hence $x \in D=L^{\eta} \cap L^{\infty}$.

It remains to show that $|x|_{D} \leqq \rho$. It is immediate that $|x|_{L^{\infty}} \leqq$ $\rho$. The argument given in the above paragraph shows that $|x|_{L^{\eta}} \leqq$ $\rho$. Thus, $|x|_{D} \leqq \rho$.

For any two functions $u, y$, (27) implies that

$$
\left\|f_{2}(t, u(t)+y(t))\right\| \leqq a(t)[\|u(t)+y(t)\|]^{o}+b(t) .
$$

Let $\rho_{1}>0$ and $\rho_{2}>0$ be given; then for $u \in \bar{S}_{D, \rho_{2}}$ and $y \in \bar{S}_{L^{\eta}, \rho_{1}}$, we assert that the right side of (28) is in $L^{\sigma}$. The Hölder inequality and the fact that $D=L^{\eta} \cap L^{\infty}$ implies that

$$
\left|f_{2}(t, u(t)+y(t))\right|_{L^{\sigma}} \leqq|a(t)|_{L^{\alpha}}\left[|u(t)|_{L^{\eta}}+|y(t)|_{L^{\eta}}\right]^{\delta}+|b(t)|_{L^{\sigma}} \cdot
$$


Since

$$
u \in \bar{S}_{D, \rho_{2}} \text { and } y \in \bar{S}_{L^{\eta}, \rho_{1}} \text { then }|u|_{D} \leqq \rho_{2} \text { and }|y|_{L^{\eta}} \leqq \rho_{1} \text {. }
$$

The result now follows from Theorem 1.

REMARK 6. If $f_{2}$ satisfies the global Lipschitz condition

$$
\left\|f_{2}\left(t, x_{1}\right)-f_{2}\left(t, x_{2}\right)\right\| \leqq a(t)\left\|x_{1}-x_{2}\right\| \quad(t \in J, x \in X)
$$

where $a \in L^{\alpha}$ and $f_{2}(t, 0) \in L^{\sigma}$ then $f_{2}$ satisfies condition (27) with $\delta=1, a=a(t)$, and $b(t)=f_{2}(t, 0)$.

REMARK 7. A sufficient condition for $\left(L^{\sigma}, L^{\eta} \cap L^{\infty}\right)$ to be admissible is that $\rho(t)$ as defined in (17) be in $L^{\eta} \cap L^{\infty}$. Indeed, for each $b \in L^{\sigma}$, the solution $w(t)$ of (5) defined by (17) satisfies the estimate

$$
\|w(t)\| \leqq|b|_{L^{\sigma}} \rho(t) \quad(t \in J) .
$$

(Related as well as supplementary comments on this topic may be found in [5].) Hence, if $\rho \in L^{\eta}(J, R)$ and (17) is satisfied, then $\left(L^{\sigma}, L^{\eta} \cap\right.$ $\left.L_{0}^{\infty}\right)$ admissible for $A(t)$.

\section{REFERENCES}

1. F. Brauer and J. S. W. Wong, On the asymptotic relationships between solutions of two systems of ordinary differential equations, J. Diff. Equations, 6 (1966), 527-543.

2. R. Conti, On the boundedness of solutions of ordinary differerential equations, Funkcialaj Ekvacioj, 9 (1966), 23-26.

3. T. G. Hallam, Asymptotic expansions in certain second order nonhomogeneous differential equations, Mathematika, 15 (1968), 30-38.

4. T. G. Hallam, On asymptotic equivalence of the bounded solutions of two systems of differential equations, Michigan Math. J., 16 (1969), 353-363.

5. - On stability and $L^{p}$ solutions of ordinary differential equations, Ann. Mat. Pura Appl., 85 (1970), 307-326.

6. P. Hartman and N. Onuchic, On the asymptotic integration of ordinary differential equations, Pacific J. Math., 13 (1963), 1193-1207.

7. J. L. Massera and J. J. Schaffer, Linear differential equations and functional analysis, IV, Mat. Ann., 139 (1960), 282-342.

8. N. Onuchic, Asymptotic relationships at infinity between the solutions of two systems of ordinary differential equations, J. Diff. Equations, 3 (1967), 47-58.

9. - Asymptotic equivalence between two systems of ordinary differential equations, Port. Mat., (to appear).

Received October 11, 1971. The research of the first author was supported in part by the National Science Foundation under grant GP-11543. It was completed while the author was visiting at the University of Rhode Island. The research of the second author was supported in part by "Conselho Nacional de Pesquisas and Fundacao de Amparo à Pesquisa do Estado de S. Paul" Brazil.

The Florida State University

AND

Universidade de São Paulo, Campus de São Carlos, Brazil 



\section{PACIFIC JOURNAL OF MATHEMATICS}

EDITORS

\author{
H. SAMELSON \\ Stanford University \\ Stanford, California 94305 \\ C. R. HOBBY \\ University of Washington \\ Seattle, Washington 98105
}

\author{
J. DUGUNDJI \\ Department of Mathematics \\ University of Southern California \\ Los Angeles, California 90007 \\ RICHARD ARENS \\ University of California \\ Los Angeles, California 90024
}

\section{ASSOCIATE EDITORS}

E. F. BECKENBACH

B. H. NeUManN

F. WOLF

K. YosHIDA

\section{SUPPORTING INSTITUTIONS}

UNIVERSITY OF BRITISH COLUMBIA

CALIFORNIA INSTITUTE OF TECHNOLOGY

UNIVERSITY OF CALIFORNIA

MONTANA STATE UNIVERSITY

UNIVERSITY OF NEVADA

NEW MEXICO STATE UNIVERSITY

OREGON STATE UNIVERSITY

UNIVERSITY OF OREGON

OSAKA UNIVERSITY
UNIVERSITY OF SOUTHERN CALIFORNIA

STANFORD UNIVERSITY

UNIVERSITY OF TOKYO

UNIVERSITY OF UTAH

WASHINGTON STATE UNIVERSITY UNIVERSITY OF WASHINGTON

AMERICAN MATHEMATICAL SOCIETY NAVAL WEAPONS CENTER 


\section{Pacific Journal of Mathematics}

\section{Vol. 45, No. $1 \quad$ September, 1973}

William George Bade, Complementation problems for the Baire classes .......... 1

Ian Douglas Brown, Representation of finitely generated nilpotent groups ........ 13

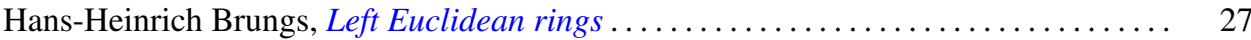

Victor P. Camillo and John Cozzens, A theorem on Noetherian hereditary rings ..... 35

James Cecil Cantrell, Codimension one embeddings of manifolds with locally flat

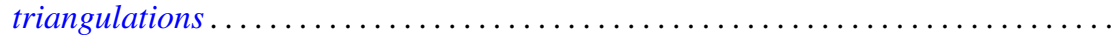

L. Carlitz, Enumeration of up-down permutations by number of rises . . . . . . . . . .

Thomas Ashland Chapman, Surgery and handle straightening in Hilbert cube

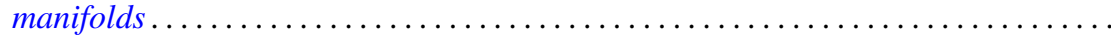

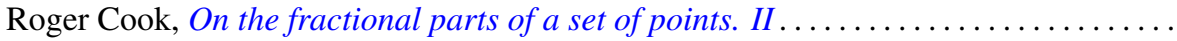

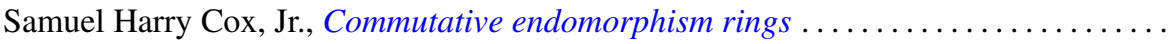

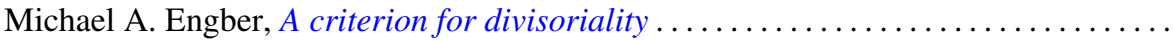

Carl Clifton Faith, When are proper cyclics injective . . . . . . . . . . . . . . 97

David Finkel, Local control and factorization of the focal subgroup . . . . . . . . . 113

Theodore William Gamelin and John Brady Garnett, Bounded approximation by

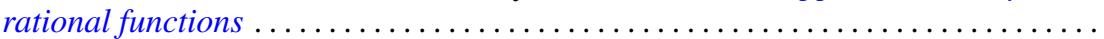

Kazimierz Goebel, On the minimal displacement of points under Lipschitzian

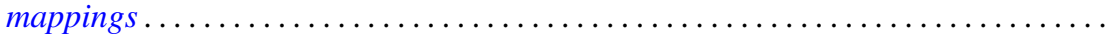

Frederick Paul Greenleaf and Martin Allen Moskowitz, Cyclic vectors for representations associated with positive definite measures: nonseparable

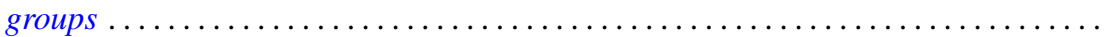

Thomas Guy Hallam and Nelson Onuchic, Asymptotic relations between perturbed linear systems of ordinary differential equations .

David Kent Harrison and Hoyt D. Warner, Infinite primes of fields and completions. .

James Michael Hornell, Divisorial complete intersections . ......

Jan W. Jaworowski, Equivariant extensions of maps ..............

John Jobe, Dendrites, dimension, and the inverse arc function .. .

Gerald William Johnson and David Lee Skoug, Feynman integrals of non-factorable

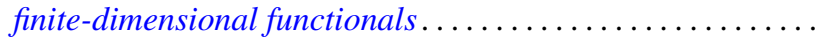

Dong S. Kim, A boundary for the algebras of bounded holomorphic functions ...... 269

Abel Klein, Renormalized products of the generalized free field and its derivatives ... 275

Joseph Michael Lambert, Simultaneous approximation and interpolation in $L_{1}$ and

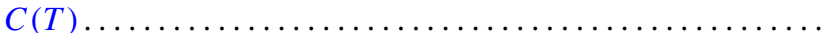

Kelly Denis McKennon, Multipliers of type $(p, p)$ and multipliers of the group

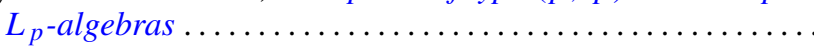

William Charles Nemitz and Thomas Paul Whaley, Varieties of implicative

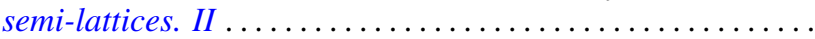

Donald Steven Passman, Some isolated subsets of infinite solvable

Norma Mary Piacun and Li Pi Su, Wallman compactifications on E-completely

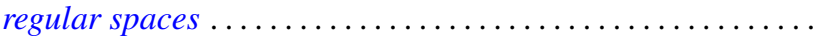

Jack Ray Porter and Charles I. Votaw, $S(\alpha)$ spaces and regular Hausdorff extensions....

Gary Sampson, Two-sided $L_{p}$ estimates of convolution transforms .

Ralph Edwin Showalter, Equations with operators forming a rig
Raymond Earl Smithson, Fixed points in partially ordered sets .

Victor Snaith and John James Ucci, Three remarks on symmetric products and

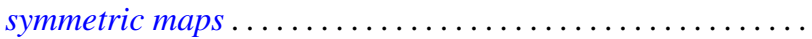

